\title{
ERRATUM
}

\section{Nanostructured materials for photon detection}

Gerasimos Konstantatos and Edward H. Sargent

Nature Nanotechnology 5, 391-400 (2010); published online: 16 May 2010; corrected after print: 6 December 2010.

In the version of this Review originally published, part of a sentence in Table 1 was missing. This error has now been corrected in the HTML and PDF versions of the text. 